\title{
Strain in wet thermally oxidized square and circular mesas
}

\author{
A. C. Alonzo, X.-C. Cheng, and T. C. McGill ${ }^{\text {a) }}$ \\ T. J. Watson Sr. Laboratory of Applied Physics, California Institute of Technology, \\ Pasadena, California 91125
}

(Received 28 June 1999; accepted for publication 4 January 2000)

\begin{abstract}
In this paper, we report the observation, through optical microscopy, of drumhead-like patterns in square and circular mesas which have been wet thermally oxidized to completion. Micro-Raman spectroscopy measurements are used to show that these patterns roughly correspond to variations in strain induced in surrounding semiconductor layers by the oxidation process. In addition, the patterns have a specific orientation with respect to the crystallographic axes of the semiconductor. A crystallographic dependence of the oxidation process itself is demonstrated and used to explain the orientation of the drumhead patterns. (C) 2000 American Institute of Physics.
\end{abstract}

[S0021-8979(00)03707-5]

\section{INTRODUCTION}

The wet thermal oxidation process has provided dramatic improvements in vertical-cavity surface-emitting laser (VCSEL) performance ${ }^{1,2}$ since its discovery in $1990 .^{3}$ VCSELs are typically fabricated with either circular or square mesas because of the large overlap between these shapes and the desired shape of the output laser beam. However, until recently, studies of the oxidation process have been concerned primarily with the simpler case of stripes. As demonstrated in several recent reports, ${ }^{4-6}$ oxidation proceeds much differently in circular mesas than in stripes. It can also be shown that the oxidation process has a unique timedependence for square mesas. ${ }^{7}$ In this paper, we explore an additional effect of geometry on the wet thermal oxidation of circular and square mesas.

Circular and square mesas, which have been completely oxidized, are shown to exhibit drumhead-like patterns, as observed with an optical microscope. We demonstrate that these drumhead patterns are related to strain induced in the semiconductor crystal by the oxidation process and that their orientation with respect to the crystallographic axes of the semiconductor is determined by the crystallographic dependence of the oxidation process itself.

This paper consists of three studies conducted to understand the drumhead patterns, their orientation, and their relationship to strain induced by the oxidation process. The first study documents the drumhead patterns and their orientation with respect to the crystallographic axes of the semiconductor. The second study uses micro-Raman spectroscopy to map the strain across the drumhead patterns by tracking the position of the GaAs LO phonon in the cap layer. The third study measures the oxidation extent in different crystallographic directions in order to identify a crystallographic dependence for the oxidation process itself.

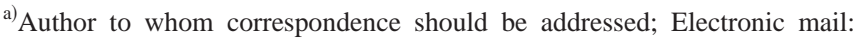
tcm@ssdp.caltech.edu

\section{DOCUMENTATION OF DRUMHEAD PATTERNS AND THEIR ORIENTATION}

\section{A. Experimental details}

The samples used in this study were grown on a (100) GaAs substrate in a Perkin Elmer MBE machine. The layer to be oxidized was $100 \mathrm{~nm}$ of AlAs, surrounded on either side by $15 \mathrm{~nm}$ of $\mathrm{Al}_{0.3} \mathrm{Ga}_{0.7}$ As. The structure was capped with $150 \mathrm{~nm}$ of GaAs. Each sample was marked with the direction of the major and minor flats to preserve knowledge of the crystal orientation. Standard photolithography procedures were used to define mesas (150 $\mu \mathrm{m}$ wide stripes, 150 $\mu \mathrm{m}$ diam. circles, or $150 \mu \mathrm{m} \times 150 \mu \mathrm{m}$ squares). These features were oriented $0^{\circ}, 45^{\circ}, 90^{\circ}$, or $135^{\circ}$ with respect to the major flat of the wafer. Hardened photoresist served as an etch mask when the samples were placed in 1:8:80 $\mathrm{H}_{2} \mathrm{SO}_{4}: \mathrm{H}_{2} \mathrm{O}_{2}: \mathrm{H}_{2} \mathrm{O}$ to remove the material around the mesas, exposing the AlAs layer. Each sample was etched immediately prior to being inserted into a $425^{\circ} \mathrm{C}$ furnace which was connected to a $95{ }^{\circ} \mathrm{C}$ water bubbler through which $1 \mathrm{l} / \mathrm{min}$ ultrapure nitrogen was flowing. In order to completely oxidize both square and circular mesas, the samples were oxidized for approximately $25 \mathrm{~min}$ under these

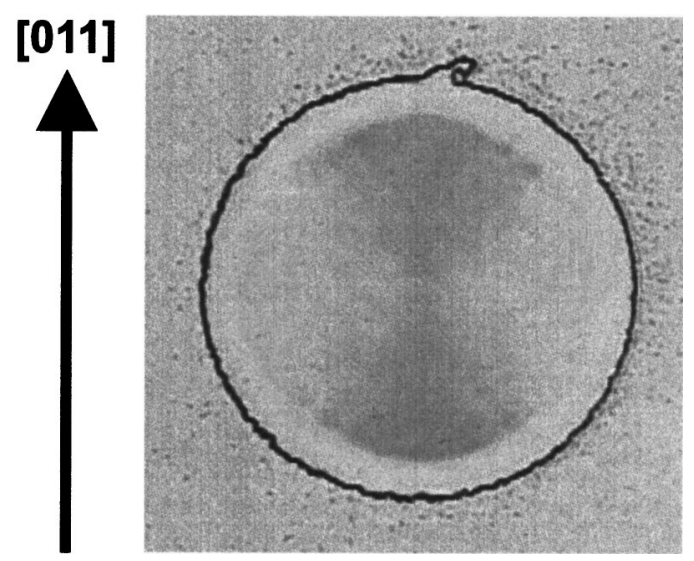

FIG. 1. Optical microscope picture of a typical circular mesa, showing the drumhead pattern. 


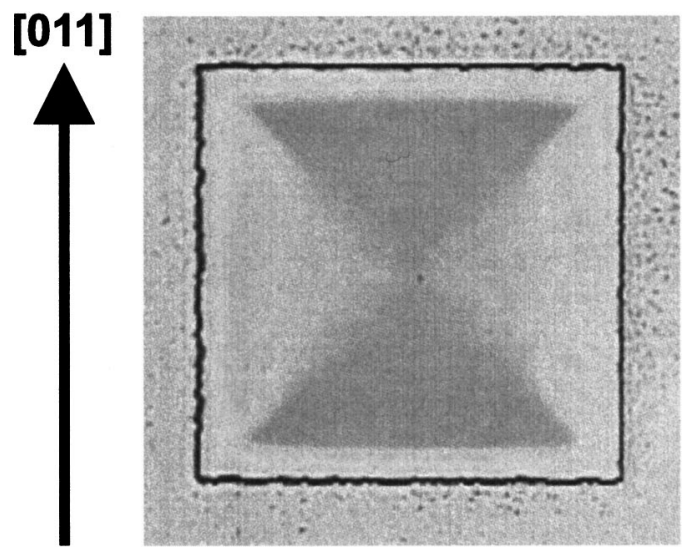

FIG. 2. Optical microscope picture of a typical square mesa aligned with the $\{011\}$ planes, showing the drumhead pattern.

conditions. An optical microscope and charge coupled device $(\mathrm{CCD})$ camera were used to obtain images of the mesas.

\section{B. Results}

Figure 1 shows a typical drumhead pattern in a circular mesa. In all four samples, this pattern was aligned along the [011] direction. For squares with sides aligned with the $\{011\}$ planes, similar drumhead patterns were observed, also aligned along the [011] direction. An example of such a square mesa is shown in Fig. 2. As shown in Fig. 3, squares with sides aligned with the $\{010\}$ planes exhibited a much different pattern, with no specific orientation with respect to the crystallographic directions.

\section{MICRO-RAMAN SPECTROSCOPY MAPPING OF DRUMHEAD PATTERNS}

\section{A. Theoretical background}

Raman spectroscopy is a useful tool for measuring strain in semiconductor crystals due to the predictable relationship between strain and shifts in the position of optical phonons. In this study, we are interested in relating the positions of GaAs optical phonons in the cap layer to strain induced by the oxidation process. This strain is believed to be the result of the volume contraction observed as the underlying AlAs layer is converted to oxide. ${ }^{8-10}$ Because the measurements were taken from the (100) face, observation of the TO modes is forbidden. ${ }^{11}$ Therefore, we are only concerned with the GaAs LO phonon. The frequency of this mode is $291.8 \mathrm{~cm}^{-1}$ in unstrained GaAs. ${ }^{12}$

A mathematical relationship between strain and phonon frequency is obtained from the following eigenvalue equation:

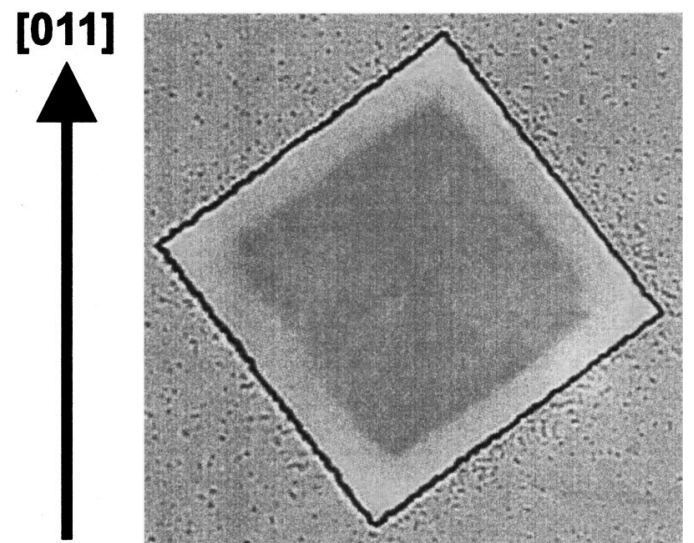

FIG. 3. Optical microscope picture of a typical square mesa aligned with the $\{010\}$ planes.

$$
\sum_{j l m} \kappa_{i j l m} \eta_{l m} u_{j}-\left(\Omega^{2}-\omega_{\circ}^{2}\right) u_{i}=0,
$$

where $\kappa_{i j l m}$ is the phonon deformation potential tensor, $\eta_{l m}$ is the strain tensor, and $\Omega$ is the unstrained frequency of the phonon. With $\lambda=\Omega^{2}-\omega_{\circ}^{2}$ and $P_{i j}=\Sigma_{l m} \kappa_{i j l m} \eta_{l m}$, Eq. (1) becomes

$$
\left|P_{i j}-\lambda \delta_{i j}\right|=0 \text {. }
$$

For a cubic crystal, there are only three independent components of the phonon deformation potential ${ }^{13}$

$$
\begin{aligned}
& p=\kappa_{1111}=\kappa_{2222}=\kappa_{3333}, \\
& q=\kappa_{1122}=\kappa_{1133}=\kappa_{2211}=\kappa_{2233}=\kappa_{3311}=\kappa_{3322}, \\
& r=\kappa_{1212}=\kappa_{1313}=\kappa_{2121}=\kappa_{2323}=\kappa_{3131}=\kappa_{3232} .
\end{aligned}
$$

Assuming that the strain is uniform in the GaAs cap layer, we expect a biaxial stress ${ }^{11,14}$

$$
\begin{aligned}
& \sigma_{y y}=\sigma_{z z}=X, \\
& \sigma_{x x}=\sigma_{x y}=\sigma_{y z}=\sigma_{z x}=0 .
\end{aligned}
$$

Under this applied (100) coplanar stress $X$, the nonzero components of the strain tensor can be written as ${ }^{14}$

$$
\eta_{\|}=\eta_{y y}=\eta_{z z}=\left(S_{11}+S_{12}\right) X,
$$

and

$$
\eta_{\perp}=\eta_{x x}=2 S_{12} X,
$$

where the $S_{i j}$ are elastic compliance constants. Therefore, Eq. (2) can be written as

$$
\left|\begin{array}{ccc}
p \eta_{\perp}+2 q \eta_{\|}-\lambda & 0 & 0 \\
0 & p \eta_{\|}+q\left(\eta_{\|}+\eta_{\perp}\right)-\lambda & 0 \\
0 & 0 & p \eta_{\|}+q\left(\eta_{\|}+\eta_{\perp}\right)-\lambda
\end{array}\right|=0
$$


for the strain induced in the GaAs cap layer by the volume contraction of an underlying oxide layer. The eigenvalues of Eq. (5) are

$$
\begin{aligned}
& \lambda_{\text {singlet }}=\lambda_{1}=p \eta_{\perp}+2 q \eta_{\|}, \\
& \lambda_{\text {doublet }}=\lambda_{2}=\lambda_{3}=p \eta_{\|}+q\left(\eta_{\|}+\eta_{\perp}\right) .
\end{aligned}
$$

Raman selection rules dictate that only the singlet mode will be detected. ${ }^{14}$ Therefore, we can write the shift in the GaAs LO phonon, $\Delta \Omega=\Omega-\omega_{\text {。 }}$, as

$$
\Delta \Omega \approx \frac{\lambda}{2 \omega_{\circ}}=\frac{p \eta_{\perp}+2 q \eta_{\|}}{2 \omega_{\circ}} .
$$

With $\eta_{\perp}=2 S_{12} /\left(S_{11}+S_{12}\right) \eta_{\|}$, Eq. (6) becomes

$$
\Delta \Omega \approx \frac{\eta_{\|}}{\omega_{\circ}}\left(\frac{p S_{12}}{S_{11}+S_{12}}+q\right) .
$$

Values for the GaAs elastic compliance constants and phonon deformation potentials have been experimentally determined $^{14}$ so that Eq. (7) becomes

$$
\Delta \Omega \approx-484 \mathrm{~cm}^{-1} \eta_{\|} .
$$

For compressive strain, $\Delta \Omega>0$; for tensile strain, $\Delta \Omega<0$.

\section{B. Experimental details}

Micro-Raman spectra were collected by Namar Scientific, Inc., using excitation from an argon-ion laser at 514.5 $\mathrm{nm}$ with a maximum power of $2 \mathrm{~mW}$, measured at the sample surface. Radiation scattered from the sample was collected in a $180^{\circ}$ backscattering geometry and directed into a Renishaw System 1000 Raman spectrometer. ${ }^{15}$ The pixel-topixel resolution of this instrument is approximately 0.5 $\mathrm{cm}^{-1}{ }^{16}$ The combined spectral resolution and reproducibility of the measurements was experimentally determined to be better than $3 \mathrm{~cm}^{-1} .15$

A Gaussian curve fit was used to precisely determine the location of the GaAs LO phonon. The form of the Gaussian profile was assumed to be

$$
f(x)=H \exp \left[-\left(\frac{x-x_{0}}{w}\right)^{2}[4 \ln (2)]\right],
$$

where $x_{0}$ is the position of the peak, $H$ is the height of the peak, and $w$ is its full width half maximum. ${ }^{15}$ The uncertainty in this fit was approximately $0.03 \mathrm{~cm}^{-1}$ for most positions on the samples. In order to determine the reliability of this technique, we examined the results of several spectroscopic scans of an unoxidized sample. After performing the Gaussian curve fits, an analysis of the variation in the fitted locations of the unstrained peak yielded an uncertainty of $0.04 \mathrm{~cm}^{-1}$. Similar uncertainty can be expected for the strained peak. Uncertainties in the measurements reported below include both the uncertainty in the fit, approximately $0.03 \mathrm{~cm}^{-1}$, and the uncertainty involved in using the Gaussian fit to determine the peak location, $0.04 \mathrm{~cm}^{-1}$. The total uncertainty in the locations of the GaAs LO phonon is approximately $0.05 \mathrm{~cm}^{-1}$ for all positions on the samples.

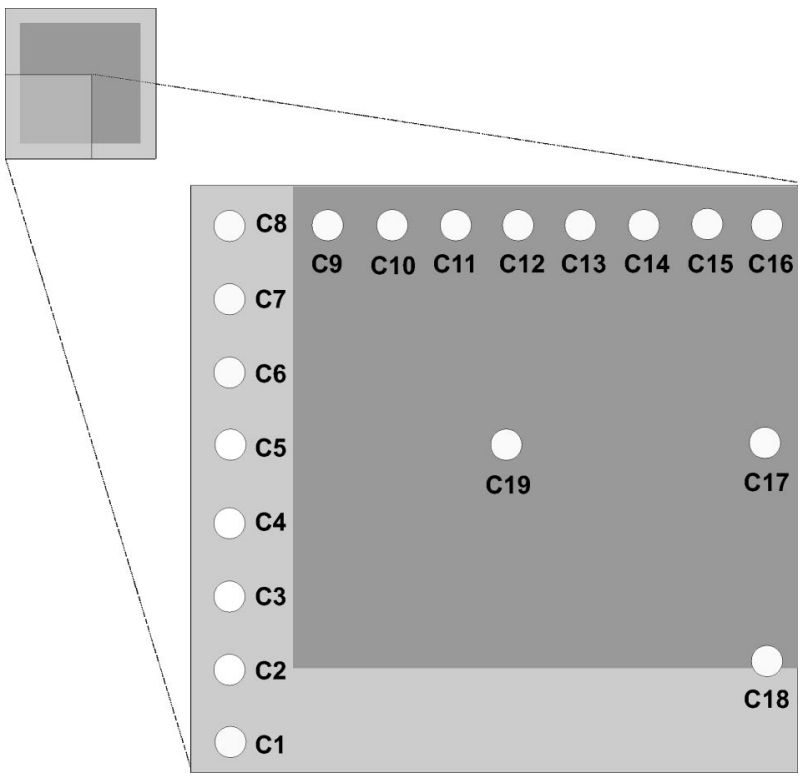

FIG. 4. Location of micro-Raman measurements on a square mesa aligned with the $\{010\}$ planes.

\section{Results}

Micro-Raman spectroscopy was used to map the position of the GaAs LO phonon across a square aligned with the $\{010\}$ planes and a square aligned with the $\{011\}$ planes, both obtained from the study described above. The locations of these measurements are shown in Figs. 4 and 5, while the phonon positions at each of these locations are listed in Tables I and II.

It is clear from the results for the square aligned with the $\{010\}$ planes that the light area of the pattern corresponds to a lower value for the position of the GaAs LO phonon than does the dark area. The average phonon position for locations C1-C8 (light area) is $291.70 \pm 0.02 \mathrm{~cm}^{-1}$, while the

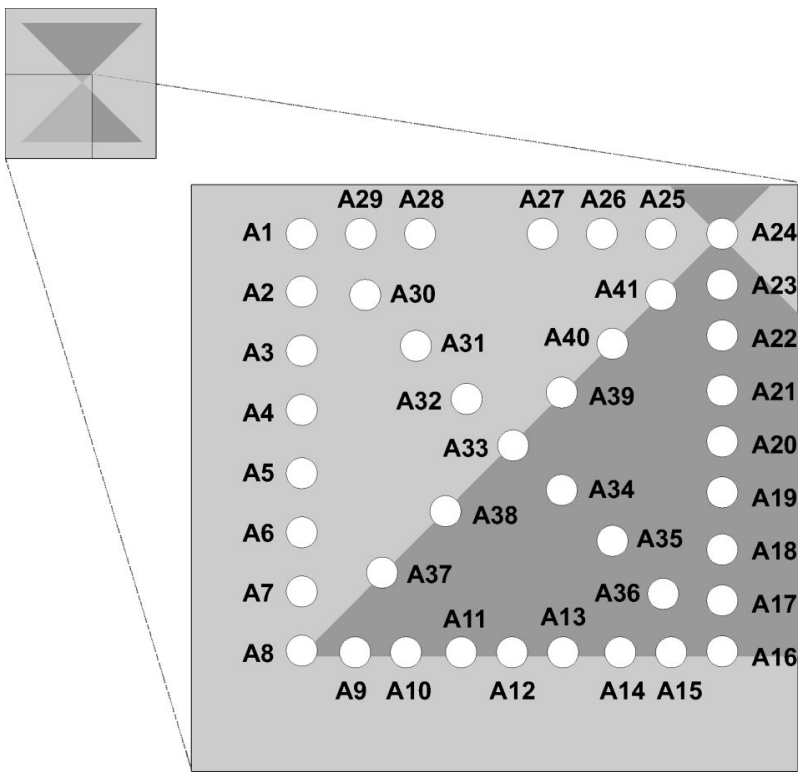

FIG. 5. Location of micro-Raman measurements on a square mesa aligned with the $\{011\}$ planes. 
TABLE I. GaAs LO phonon positions in a square mesa aligned with the $\{010\}$ planes.

\begin{tabular}{|c|c|}
\hline Measurement location & GaAs LO phonon position $\left(\mathrm{cm}^{-1}\right)$ \\
\hline $\mathrm{C} 1$ & $291.71 \pm 0.05$ \\
\hline $\mathrm{C} 2$ & $291.68 \pm 0.05$ \\
\hline $\mathrm{C} 3$ & $291.71 \pm 0.05$ \\
\hline $\mathrm{C} 4$ & $291.70 \pm 0.05$ \\
\hline $\mathrm{C} 5$ & $291.70 \pm 0.05$ \\
\hline C6 & $291.72 \pm 0.05$ \\
\hline $\mathrm{C} 7$ & $291.71 \pm 0.05$ \\
\hline $\mathrm{C} 8$ & $291.71 \pm 0.05$ \\
\hline C9 & $292.10 \pm 0.05$ \\
\hline $\mathrm{C} 10$ & $292.19 \pm 0.05$ \\
\hline $\mathrm{C} 11$ & $292.21 \pm 0.05$ \\
\hline $\mathrm{C} 12$ & $292.18 \pm 0.05$ \\
\hline $\mathrm{C} 13$ & $292.18 \pm 0.05$ \\
\hline $\mathrm{C} 14$ & $292.17 \pm 0.05$ \\
\hline $\mathrm{C} 15$ & $292.15 \pm 0.05$ \\
\hline $\mathrm{C} 16$ & $292.15 \pm 0.05$ \\
\hline $\mathrm{C} 17$ & $292.19 \pm 0.05$ \\
\hline $\mathrm{C} 18$ & $292.98 \pm 0.05$ \\
\hline C19 & $293.17 \pm 0.05$ \\
\hline
\end{tabular}

average for locations C9-C17 (dark area) is 292.17 \pm 0.02 $\mathrm{cm}^{-1}$. Note that the latter average excludes $\mathrm{C} 19$, the measurement taken at the center of the dark area. At 293.17 $\pm 0.05 \mathrm{~cm}^{-1}$, the position of the GaAs LO phonon at this point is significantly higher than the positions measured at locations $\mathrm{C} 9-\mathrm{C} 17$. The measurement at location $\mathrm{C} 18$ is also excluded from both averages, since it lies on the boundary between the dark and light areas.

Similar results are obtained for the square aligned with the $\{011\}$ planes. Again, the position of the GaAs LO phonon in the light region is generally observed at a lower wave number than in the dark region. Measurements taken in the center of the sampled area have an average GaAs LO phonon position of $289.85 \pm 0.03 \mathrm{~cm}^{-1}$ in the light region (A30A32) and $290.01 \pm 0.03 \mathrm{~cm}^{-1}$ in the dark region (A34-A36). Similarly, a comparison can be made between measured phonon positions along the [011] direction: $289.85 \pm 0.02 \mathrm{~cm}^{-1}$ in the light region (A2-A7) and 290.16 $\pm 0.02 \mathrm{~cm}^{-1}$ in the dark region (A17-A23). In order to obtain a more complete picture of the GaAs LO phonon position in this mesa, Fig. 6 shows a three-dimensional representation of the microRaman data. The $x$ - and $y$-axes correspond to the spatial dimensions of the mesa, while the $z$-axis shows the position of the GaAs LO phonon at each spatial location. Light and dark shading indicate the region of the mesa from which each measurement was taken. In addition to the observations above, it can be seen that the GaAs LO phonon is at a significantly higher position for measurements taken along the [01] direction, as compared to those taken along the [011] direction.

\section{Conclusions}

As shown above, there is a rough correspondence between the light and dark regions of the drumhead patterns
TABLE II. GaAs LO phonon positions in a square mesa aligned with the $\{011\}$ planes.

\begin{tabular}{lc}
\hline \hline Measurement location & GaAs LO phonon position $\left(\mathrm{cm}^{-1}\right)$ \\
\hline A1 & $291.12 \pm 0.05$ \\
A2 & $289.91 \pm 0.05$ \\
A3 & $289.89 \pm 0.05$ \\
A4 & $289.76 \pm 0.05$ \\
A5 & $289.86 \pm 0.05$ \\
A6 & $289.83 \pm 0.05$ \\
A7 & $289.81 \pm 0.05$ \\
A8 & $291.12 \pm 0.05$ \\
A9 & $290.94 \pm 0.05$ \\
A10 & $291.02 \pm 0.05$ \\
A11 & $291.09 \pm 0.05$ \\
A12 & $291.26 \pm 0.05$ \\
A13 & $291.15 \pm 0.05$ \\
A14 & $291.42 \pm 0.05$ \\
A15 & $291.10 \pm 0.05$ \\
A16 & $291.19 \pm 0.05$ \\
A17 & $290.14 \pm 0.05$ \\
A18 & $290.16 \pm 0.05$ \\
A19 & $290.17 \pm 0.05$ \\
A20 & $290.14 \pm 0.05$ \\
A21 & $290.15 \pm 0.05$ \\
A22 & $290.21 \pm 0.05$ \\
A23 & $290.14 \pm 0.05$ \\
A24 & $290.19 \pm 0.05$ \\
A25 & $291.20 \pm 0.05$ \\
A26 & $291.13 \pm 0.05$ \\
A27 & $291.11 \pm 0.05$ \\
A28 & $291.10 \pm 0.05$ \\
A29 & $291.17 \pm 0.05$ \\
A30 & $289.85 \pm 0.05$ \\
A31 & $289.79 \pm 0.05$ \\
A32 & $289.92 \pm 0.05$ \\
A33 & $291.12 \pm 0.05$ \\
A34 & $290.02 \pm 0.05$ \\
A35 & $290.05 \pm 0.05$ \\
A36 & $289.93 \pm 0.05$ \\
A37 & $290.00 \pm 0.05$ \\
A38 $3401.08 \pm 0.05$ \\
A41 & $291.04 \pm 0.05$ \\
& \\
\hline
\end{tabular}

and the position of the GaAs LO phonon measured in those regions. In general, the GaAs LO phonon is observed at a lower wave number in the light regions, as compared to its position in the dark regions. Because of unequal heating in oxidized and unoxidized samples, ${ }^{11}$ we cannot make a direct comparison of phonon positions in our samples before and after oxidation. However, we can obtain an estimate of the strain induced in the samples by the oxidation process by assuming an unstrained GaAs LO phonon position of 291.8 $\mathrm{cm}^{-1} \cdot{ }^{12}$ For simplicity, we obtain this estimate by considering the phonon positions measured in the square aligned with the $\{010\}$ directions. The average phonon shift in the light region is $-0.10 \pm 0.02 \mathrm{~cm}^{-1}$, while the average phonon shift in the dark region is $+0.37 \pm 0.02 \mathrm{~cm}^{-1}$. This corresponds to a tensile strain of $2.1 \pm 0.4 \times 10^{-4}$ in the light region and a compressive strain of $7.6 \pm 0.4 \times 10^{-4}$ in the dark region. 


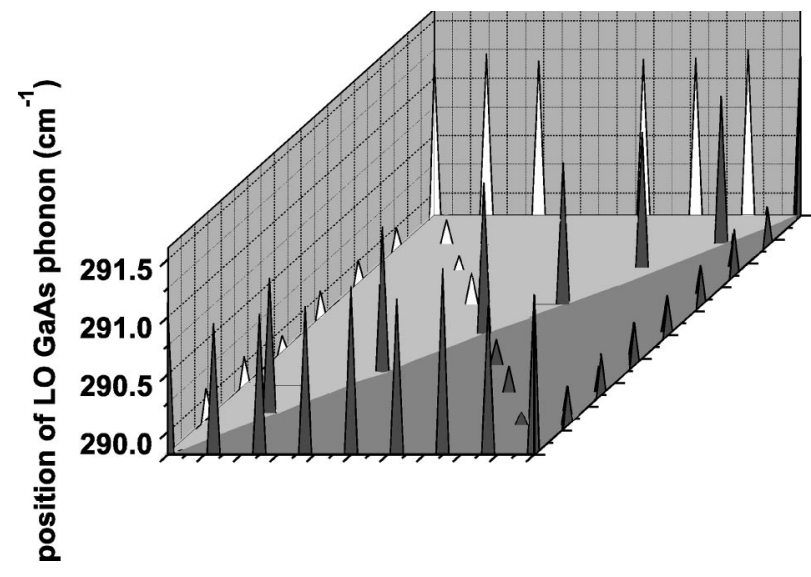

FIG. 6. Three-dimensional representation of the micro-Raman measurements for a square mesa aligned with the $\{011\}$ planes. The $x$ - and $y$-axes correspond to the spatial dimensions of the mesa, while the $z$-axis shows the position of the GaAs LO phonon at each spatial location. The light and dark shading corresponds to the shading of the mesa at each location.

\section{CRYSTALLOGRAPHIC DEPENDENCE OF THE OXIDATION PROCESS}

\section{A. Experimental details}

The samples used in this study were processed in an identical manner to those in the first study, except that each was oxidized for precisely $12 \mathrm{~min}$. Two optical microscope pictures were taken and measured for each mesa. The images were oriented $90^{\circ}$ with respect to each other, in order to ensure that any distortion which might exist in the CCD image would not influence the measurements. For each mesa, the oxidation extent was obtained by measuring the length of oxidation in each direction parallel to the sides of the aperture.

\section{B. Results}

Partially oxidized stripes aligned with the [011], [011] [001], and [010] directions provide the most straightforward comparison of the oxidation rates. Table III shows the extent of oxide formed in the stripes on each of the four samples. It can be concluded from these data that oxidation along the [011] direction is slightly faster than along the [011] direction. Within the experimental uncertainty, there is no difference in the oxidation rates observed along the $\langle 010\rangle$ directions and between the rates along these directions and the [011] direction. Therefore, only the [011] direction is observed to have a reduced oxidation rate.

As shown in Table IV, the results obtained for circular mesas show that the oxidized apertures are quite symmetric.

TABLE III. Extent of oxide formed in stripes after 12 min wet thermal oxidation at $425^{\circ} \mathrm{C}$.

\begin{tabular}{ccc}
\hline \hline Sample & Direction of oxidation & Oxide extent $(\mu \mathrm{m})$ \\
\hline A & {$[011]$} & $50.7 \pm 0.4$ \\
B & {$[011]$} & $47 \pm 2$ \\
C & {$[001]$} & $50 \pm 1$ \\
D & {$[010]$} & $51 \pm 2$ \\
\hline \hline
\end{tabular}

TABLE IV. Extent of oxide formed in $150 \mu \mathrm{m}$ diam circular mesas after 12 min wet thermal oxidation at $425^{\circ} \mathrm{C}$.

\begin{tabular}{ccc}
\hline \hline Sample & {$[010]$ oxide extent $(\mu \mathrm{m})$} & {$[001]$ oxide extent $(\mu \mathrm{m})$} \\
\hline A & $65.9 \pm 0.5$ & $66.1 \pm 0.7$ \\
B & $64 \pm 2$ & $64 \pm 2$ \\
C & $62 \pm 2$ & $62 \pm 3$ \\
D & $62 \pm 4$ & $62 \pm 4$ \\
\hline \hline
\end{tabular}

Within experimental uncertainty, identical oxidation extents are obtained in the [001] and [010] directions for a given sample. All of the circular mesas in this study oxidized to produce diamond-shaped apertures with sides aligned along the $\langle 010\rangle$ crystal axes. The consistency in the orientation of the apertures seems to support a crystallographic preference for the oxidation process.

The oxidation of squares (Table V) is consistent with the results for both stripes and circles. Oxidation along the [010] and [001] directions is virtually identical. Although the results from sample B show identical oxidation in the [011] and $[01 \overline{1}]$ directions, those from sample $\mathrm{A}$ indicate a slight difference between these two directions. In agreement with the results obtained for stripes, oxidation in the [011] direction appears to proceed slightly faster than in the [011] direction. It should be noted that, in the case of the square mesas, this conclusion is dependent upon the lower uncertainty of the oxide extents measured in sample A. We believe that this difference is a real effect and can be attributed to the smaller size of sample A, and thus a reduction in variation across the sample.

\section{Conclusions}

Our data show a slight reduction in oxidation rate for the [011] direction, as compared to the [011] and $\langle 010\rangle$ directions. Within the experimental uncertainty of our measurements, there is no difference in oxidation rates for the [011] and $\langle 010\rangle$ directions. This is consistent with the orientation of diamond-shaped apertures in the circular mesas since this allows for a slower oxidation rate in the $[01 \overline{1}]$ direction.

Based on the shape and orientation of the apertures in circular mesas, Choquette et al. predict a slightly faster oxidation along the $\langle 010\rangle$ crystal axes, as compared to that along the $\langle 011\rangle$ axes. ${ }^{17,18}$ Although our data support a faster oxidation in the $\langle 010\rangle$ directions than in the [011] direction, the results obtained in this study do not follow the more generalized prediction of slower oxidation in the $\langle 011\rangle$ directions. However, Li et al. predict a different oxidation pro-

TABLE V. Extent of oxide formed in $150 \mu \mathrm{m} \times 150 \mu \mathrm{m}$ square mesas after 12 min wet thermal oxidation at $425^{\circ} \mathrm{C}$.

\begin{tabular}{ccc}
\hline \hline Sample & {$[011]$ oxide extent $(\mu \mathrm{m})$} & {$[01 \overline{1}]$ oxide extent $(\mu \mathrm{m})$} \\
\hline A & $56.0 \pm 0.9$ & $54.4 \pm 0.8$ \\
B & $54 \pm 2$ & $54 \pm 3$ \\
Sample & {$[010]$ oxide extent $(\mu \mathrm{m})$} & {$[001]$ oxide extent $(\mu \mathrm{m})$} \\
\hline C & $54 \pm 2$ & $54 \pm 2$ \\
D & $54 \pm 4$ & $54 \pm 4$ \\
\hline \hline
\end{tabular}


file along the [011] and [01 $\overline{1}]$ directions because of the excellent polarization selection and control observed in oxideconfined VCSELs. ${ }^{19}$ Our results support both this prediction and, as will be discussed below, the observations and measurements reported earlier in this paper.

\section{CONCLUSIONS}

Based on the results reported above, it seems that the drumhead patterns observed in circular and square mesas indicate variations in strain induced in the semiconductor crystal by the oxidation process. In addition, the crystallographic dependence of the oxidation process appears to be responsible for the consistent orientation of these patterns with respect to the crystallographic axes of the semiconductor. If the oxidation had no crystallographic dependence, we would not expect the drumhead pattern to have a specific crystallographic orientation. However, because the [011] direction has a different oxidation rate, this deviation ensures that the drumhead pattern will have a specific orientation with respect to this direction.

The volume contraction of the AlAs layer upon oxidation is well known and is believed to be responsible for strain induced in the surrounding semiconductor crystal by the oxidation process. ${ }^{8-10}$ As demonstrated by Landesman et al., the oxidation process induces local strain in the GaAs cap layer at the oxide-AlAs interface. ${ }^{11}$ Because of the reduced oxidation rate in the $[01 \overline{1}]$ direction, the volume contraction of the oxide will not be symmetric with respect to the center of the mesa. This effect should be greatest as the oxidation nears completion and may explain the drumhead patterns observed in mesas, which are almost completely oxidized. However, the exact mechanisms involved in the formation of the drumhead patterns are not completely understood at the present time.

\section{ACKNOWLEDGMENTS}

The micro-Raman spectroscopy results reported in this paper were taken by F. Hardcastle at Namar Scientific, Inc. The work was supported in part by the Office of Naval Research under Grant No. N00014-98-1-0567.

${ }^{1}$ K. Lear, K. Choquette, R. Schneider, Jr., S. Kilcoyne, and K. Geib, Electron. Lett. 31, 208 (1995).

${ }^{2}$ G. Yang, M. MacDougal, and P. Dapkus, Electron. Lett. 31, 886 (1995).

${ }^{3}$ J. Dallesasse, N. Holonyak, Jr., A. Sugg, T. Richard, and N. El-Zein, Appl. Phys. Lett. 57, 2844 (1990).

${ }^{4}$ B. Koley, M. Dagenais, R. Jin, J. Pham, G. Simonis, G. McLane, and D. Stone, J. Appl. Phys. 82, 4586 (1997).

${ }^{5}$ T. Langenfelder, S. Schröder, and H. Grothe, J. Appl. Phys. 82, 3548 (1997).

${ }^{6}$ A. Alonzo, X.-C. Cheng, and T. McGill, J. Appl. Phys. 84, 6901 (1998).

${ }^{7}$ A. Alonzo, X.-C. Cheng, and T. McGill (unpublished, 1998).

${ }^{8}$ T. Takamori, K. Takemasa, and T. Kamijoh, Appl. Phys. Lett. 69, 659 (1996).

${ }^{9}$ D. Deppe, D. Huffaker, T.-H. Oh, H. Deng, and Q. Deng, IEEE J. Sel. Top. Quantum Electron. 3, 893 (1997).

${ }^{10}$ J. Seo and K. Seo, Appl. Phys. Lett. 72, 1466 (1998).

${ }^{11}$ J. Landesman, A. Fiore, J. Nagle, V. Berger, E. Rosencher, and P. Puech, Appl. Phys. Lett. 71, 2520 (1997).

${ }^{12}$ A. Sood, E. Anastassakis, and M. Cardona, Phys. Status Solidi B 129, 505 (1985).

${ }^{13}$ E. Anastassakis, A. Pinczuk, E. Burstein, F. Pollack, and M. Cardona, Solid State Commun. 8, 133 (1970).

${ }^{14}$ G. Landa, R. Carles, C. Fontaine, E. Bedel, and A. Muñoz-Yagüe, J. Appl. Phys. 66, 196 (1989).

${ }^{15}$ F. Hardcastle, Technical Report No. 98-11005, Namar Scientific, Inc., 1998.

${ }^{16} \mathrm{~F}$. Hardcastle (private communication, 1999).

${ }^{17}$ K. Choquette, K. Geib, H. Chui, B. Hammons, H. Hou, T. Drummond, and R. Hull, Appl. Phys. Lett. 69, 1385 (1996).

${ }^{18}$ K. Choquette et al., IEEE J. Sel. Top. Quantum Electron. 3, 916 (1997).

${ }^{19}$ G. Li, S. Lim, W. Yuen, and C. Chang-Hasnain, Electron. Lett. 31, 2014 (1995). 